\title{
Surgical management of spinal meningiomas: focus on unilateral posterior approach and anterior localization
}

\author{
Julia Onken, MD, ${ }^{1,2}$ Kathrin Obermüller, MD, ${ }^{3}$ Franziska Staub-Bartelt, MD, ${ }^{1}$ Bernhard Meyer, MD, ${ }^{3}$ \\ Peter Vajkoczy, MD, ${ }^{1}$ and Maria Wostrack, MD ${ }^{3}$
}

\begin{abstract}
'Department of Neurosurgery, Charité-Universitätsmedizin Berlin; 'Berlin School of Integrative Oncology (BSIO), Berlin; and ${ }^{3}$ Department of Neurosurgery, Klinikum rechts der Isar, Technical University of Munich, Germany
\end{abstract}

\begin{abstract}
OBJECTIVE Spinal meningiomas (sMNGs) are relatively rare in comparison to intracranial MNGs. sMNGs localized anterior to the denticulate ligament (aMNGs) represent a surgically challenging subgroup. A high perioperative complication rate due to the need for complex surgical approaches has been described. In the present study, the authors report on their surgical experience that involves two institutions in which 207 patients underwent surgery for sMNGs. Special focus was placed on patients with aMNGs that were treated via a unilateral posterior approach (ULPA).

METHODS Between 2005 and 2017, 207 patients underwent resection of sMNGs at one of two institutions. The following characteristics were assessed: tumor size and localization, surgical approach, duration of surgery, grade of resection, peri- and postoperative complication rates, and neurological outcome. Data were compared between the subgroups of patients according to the lesion's relationship to the denticulate ligament and to surgical approach.

RESULTS The authors identified 48 patients with aMNGs, 86 patients with lateral MNGs, and 76 patients with posterior MNGs (pMNGs). Overall, $66.6 \%$ of aMNGs and $64 \%$ of pMNGs were reached via a ULPA. aMNGs that were approached via a ULPA showed reduced duration of surgery (131 vs 224 minutes, $p<0.0001$ ) and had surgical complication rates and neurological outcomes comparable to those of lesions that were approached via a bilateral approach. No significant differences in complication rate, outcomes, and extent of resection were seen between aMNGs and pMNGs.

CONCLUSIONS The duration of surgery, extent of resection, and outcomes are comparable between aMNGs and pMNGs when removed via a ULPA. Thus, ULPA represents a safe route to achieve a gross-total resection, even in cases of aMNG.
\end{abstract}

https://thejns.org/doi/abs/10.3171/2018.8.SPINE18198

KEYWORDS spinal meningioma; hemilaminectomy; denticulate ligament; oncology

$\mathrm{T}$ HE incidence of spinal meningiomas (sMNGs) is relatively low when compared to that of intracranial MNGs. ${ }^{10}$ sMNGs account for approximately $1.2 \%$ of all MNGs of the central nervous system. sMNGs are mainly localized in the thoracic spine (range 67\%-84\%); far fewer are seen in the cervical spine (range 14\%-27\%), and fewer still are localized in the lumbar spine (range $2 \%-14 \%) .{ }^{5,14}$ Because of its association with improved neurological outcome and low recurrence rate, surgical treatment of sMNGs remains the gold standard., 2,67 The literature indicates that gross-total resection (GTR) is achieved in $82 \%-99 \%$ of cases. ${ }^{37,14,16}$ In these publications, GTR is defined as tumor removal without excision of its dural at- tachment and with the dura electrically coagulated only, according to a Simpson grade II resection..$^{15,19,21}$ In contrast to cranial MNGs, sMNGs have recurrence rates that range merely from $1.3 \%$ to $6.4 \%$ after a Simpson grade II resection has been achieved, and hence the procedure seems sufficient to control recurrence rate. ${ }^{16,19}$

To achieve GTR, different surgical approaches have been applied and depend on tumor localization and size. sMNGs anterior to the denticulate ligament (aMNGs) represent a surgically challenging subgroup due to difficult surgical exposure, and thus increased peri- and postoperative complication rates have been discussed for this subgroup of lesions..$^{13,17,18}$ As a consequence, recent articles

ABBREVIATIONS aMNG = anterior meningioma; BLPA = bilateral posterior approach; GTR = gross-total resection; KPS = Karnofsky Performance Scale; pMNG = posterior meningioma; sMNG = spinal meningioma; ULPA = unilateral posterior approach.

SUBMITTED February 18, 2018. ACCEPTED August 6, 2018.

INCLUDE WHEN CITING Published online December 7, 2018; DOI: 10.3171/2018.8.SPINE18198. 
TABLE 1. Summary of patient characteristics

\begin{tabular}{|c|c|c|c|}
\hline Characteristic & aMNG & pMNG & $p$ Value \\
\hline $\begin{array}{l}\text { Localization of meningioma to } \\
\text { denticulate ligament, no. }\end{array}$ & $48(23 \%)$ & $76(37 \%)$ & NS \\
\hline Mean age at diagnosis (range), yrs & $64(29-86)$ & $64(19-87)$ & NS \\
\hline Female/male ratio & $6.25: 1$ & $3.2: 1$ & NS \\
\hline \multicolumn{4}{|l|}{ Duration of symptoms } \\
\hline $1-12$ wks & $20 \%$ & $28 \%$ & NS \\
\hline $3-12$ mos & $60 \%$ & $56 \%$ & NS \\
\hline$>1 \mathrm{yr}$ & $20 \%$ & $16 \%$ & NS \\
\hline \multicolumn{4}{|l|}{ Clinical presentation } \\
\hline Pain & $17 \%$ & $57 \%$ & $<0.0001$ \\
\hline Motor deficit & $38 \%$ & $25 \%$ & NS \\
\hline Sensory deficit & $47 \%$ & $46 \%$ & NS \\
\hline Long tract impairment & $43 \%$ & $18 \%$ & 0.0002 \\
\hline Vegetative dysfunction & $19 \%$ & $31 \%$ & NS \\
\hline Mean preop KPS score & $90 \%$ & $90 \%$ & NS \\
\hline WHO grade I MNG & $93 \%$ & $94 \%$ & NS \\
\hline WHO grade II MNG & $7 \%$ & $6 \%$ & NS \\
\hline Mean hospital stay, days & 10 & 9 & NS \\
\hline Follow-up 1 yr postop & \multicolumn{2}{|c|}{$73 \%$} & - \\
\hline Mean follow-up duration (range) & \multicolumn{2}{|c|}{14 mos (3 mos to $7 \mathrm{yrs}$ ) } & - \\
\hline
\end{tabular}

NS = not significant; $-=$ not applicable.

involving aMNGs have discussed anterior approaches that involve a single-level or multilevel vertebrectomy and placement of instrumentation; anterior approaches are more complex in nature than traditional posterior approaches. ${ }^{9}, 12,13$ These studies reported a complication rate for aMNG of up to $27 \%$, mostly approach related. ${ }^{9}$ In contrast, only a few studies with small sample sizes have reported on accessing sMNGs from a unilateral posterior approach (ULPA). ${ }^{12}$ Here, we report on our surgical experience with 207 cases of sMNG treated at two institutions. We compared surgical techniques, complication rates, and outcome in the treatment of sMNGs according to their location in relationship to the denticulate ligament. We show that MNGs, even when localized anterior to the denticulate ligament, can be safely approached and successfully removed via a ULPA. More extended posterior or even anterior approaches for aMNG are rarely indicated but were chosen in cases of extensive calcifications in combination with myelopathy demonstrated on preoperative MRI.

\section{Methods \\ Patient Data}

Between 2005 and 2017, 207 patients with sMNGs underwent resection at one of two institutions. We retrospectively collected data on demographics, symptoms at clinical presentation, Karnofsky Performance Scale (KPS) score, neurological status according to McCormick score, and onset of symptoms. Radiological findings included exact localization of the MNG in the spinal canal in relation to the denticulate ligament. MNGs localized anterior or anterolateral to the denticulate ligament were labeled aMNGs. Those localized posterior or posterolateral to the dentate ligament were termed "posterior meningiomas"
(pMNGs). The term "lateral MNG" was used for lesions that could not be assigned to either the aMNG group or pMNG group. Lateral MNGs were not included in the analysis. Size was given in longitudinal, sagittal, and axial diameter in millimeters. The percentage that the lesion occupied the spinal canal on axial MRI scans was also determined. Furthermore, the grade of tumor resection according to Simpson scheme, duration of surgery, and periand postoperative surgical and neurological complications were assessed. The resection grade was determined with the help of the surgical report and postoperative MRI studies. Outcome data included neurological function (McCormick score), KPS score, and MRI studies obtained during outpatient follow-up. Detailed patient characteristics are given in Table 1.

\section{Surgical Technique}

The majority of tumors were removed via a ULPA. A bilateral posterior approach (BLPA) included laminectomy or laminoplasty. The ULPA included hemilaminectomy and (extended) interlaminar fenestration. A small skin incision of $4 \mathrm{~cm}$ (single-level approach) was used. A tissuesparing retractor system was inserted. In the ULPA, bony removal was limited to the unilateral lamina at a corridor of $1 \mathrm{~cm}$. The surgical field was enlarged by completely removing the ligamentum flavum and undercutting of the contralateral side. After making a longitudinal incision in the dura, the dura was sutured in place medially and laterally. In cases of extensive MNGs, the given corridor was used to access the tumor from its ipsilateral expansion. The tumor was debulked with suction and a dissector, and its dural attachment was coagulated. In cases of small and purely anterior MNGs, tumor exposure was achieved via myelon rotation. The denticulate ligament was cut from its dural attachment and drawn medially with a suture. In cases of extensive tumors of the thoracic spine, the denticulate ligament and nerve roots were cut to achieve sufficient rotation of the myelon and tumor exposure. A BLPA, or an even more extended approach, was rare at either institution, but it was mostly chosen in cases involving tumor calcifications in combination with myelopathy demonstrated on preoperative MRI scans.

Surgical procedures were carried out by 5-7 neurosurgeons per institution. The experience level of the surgeons was comparable at each institution. Surgeons who passed residency 1-5 years ago carried out one-third of the procedures, and two-thirds were performed by more experienced surgeons.

\section{Histological Examination}

Formalin-fixed paraffin-embedded sections were obtained for histological evaluation. The neuropathological department of each hospital provided histological confirmation of the diagnosis according to the WHO classification.

\section{Statistical Analysis}

Statistical calculations were performed using GraphPad Prism 5.0c (GraphPad Software Inc.) and Microsoft Excel 2016. Results were analyzed using the Student t-test calculating means and standard deviations. Chi-square 

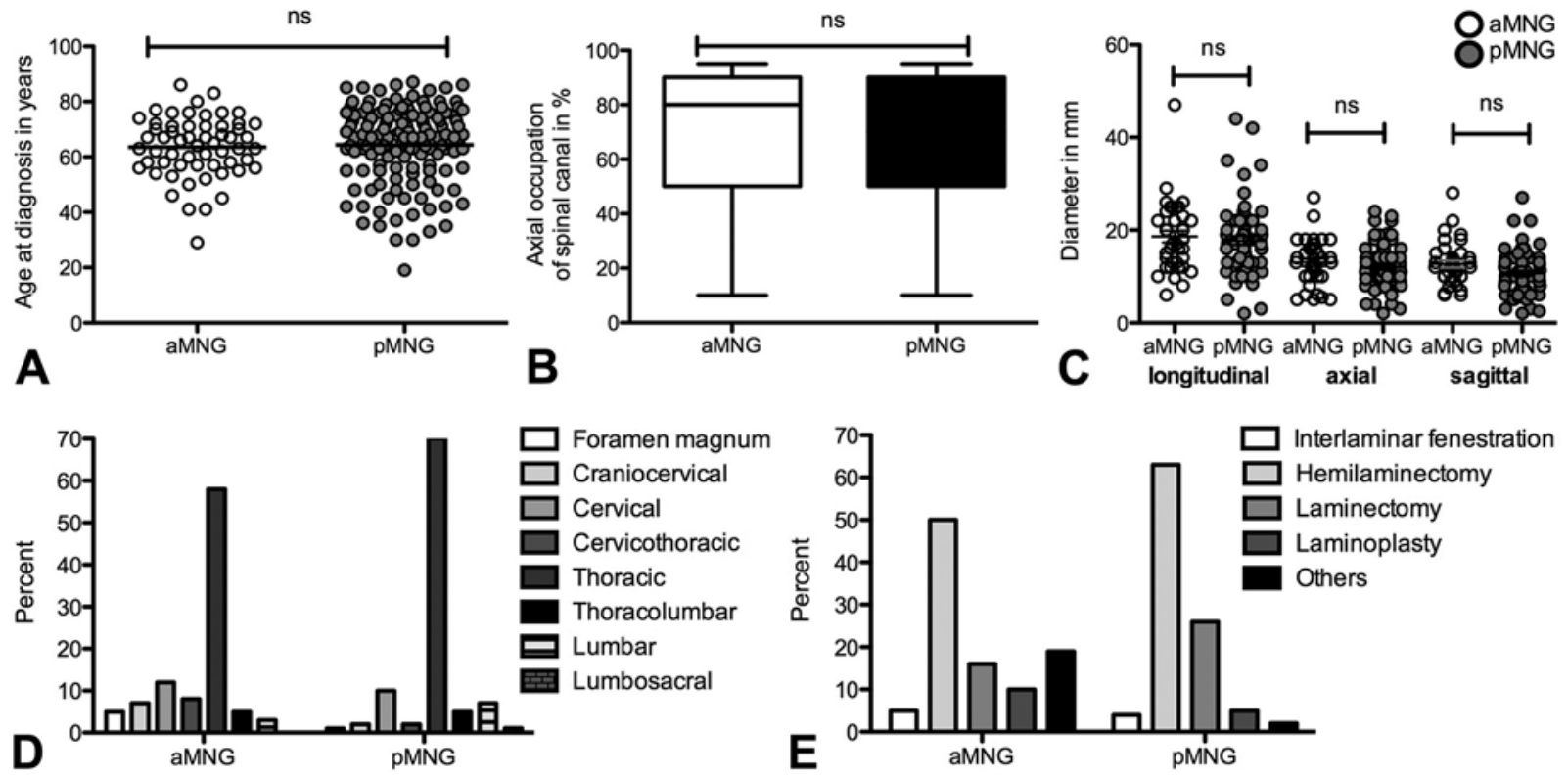

FIG. 1. A: Mean age at diagnosis of an sMNG depending on the lesion's localization within the spinal canal. B: Percentage of axial spinal canal occupied by the MNG. C: Size of sMNG in terms of longitudinal, sagittal, and axial diameter. D: Distribution of sMNGs within the spinal canal and according to the denticulate ligament. E: Surgical approaches, which were used for different MNG localizations. ns $=$ not significant.

and Fisher exact tests were used for analysis of contingency tables. The one-way ANOVA Bonferroni multiple comparison test was used to compare differences between groups. The level of significance was set at $\mathrm{p}<0.05$.

All procedures performed in studies involving human participants were in accordance with the ethical standards of the institutional research committee and with the 1964 Helsinki Declaration and its later amendments or comparable ethical standards. For this type of study, formal consent is not required. The local ethics committee approved the study design.

\section{Results \\ Clinical Data}

We identified 48 patients with aMNGs, 86 patients with lateral MNGs, and 76 patients with pMNGs. The mean age at diagnosis was 65 years, and the study population was $81 \%$ female. Follow-up durations varied from 3 months to 7 years (median 14 months). Overall, 27\% of patients were lost to follow-up 1 year after surgery. Follow-up was conducted in outpatient clinics of both institutions with MRI at 3 and 12 months after surgery and then routinely every 12-24 months thereafter. Age did not differ significantly between patients with aMNG and those with pMNG (Fig. 1A). At diagnosis, the mean tumor diameters were 18, 12, and $12 \mathrm{~mm}$ in the longitudinal, sagittal, and axial planes. Comparing aMNGs and pMNGs, no significant difference in size was found (Fig. 1B). The mean axial involvement of the spinal canal was approximately 65\% (range 10\%95\%). aMNGs and pMNGs did not differ significantly in axial involvement of the spinal canal (Fig. 1C). sMNGs were localized predominantly in the thoracic spine (Fig. 1D). Surgical exposure of sMNG was exclusively achieved via a dorsal approach, using either the ULPA or the BLPA
(Fig. 1E). The most common symptoms in patients with aMNGs were sensory deficits (47\%), long tract signs (43\%), and motor deficits (38\%). The most common symptoms in patients with pMNGs were pain (57\%), sensory deficits $(46 \%)$, motor deficits (25\%), and long tract signs (18\%). The duration of symptoms prior to clinical presentation varied from 1 to 12 weeks (25\%), 3 to 12 months (56\%), and > 1 year $(18 \%)$. According to MNG localization, symptom duration until diagnosis was comparable between patients with an aMNG (1-12 weeks in 20\%, 3-12 months in 60\%, $>1$ year in 20\%) and those with a pMNG (1-12 weeks in $28 \%, 3-12$ months in 56\%, > 1 year in $16 \%$ ). Among the 207 patients, $97 \%$ of the MNGs were WHO grade I and $3 \%$ were atypical MNGs of WHO grade II. Detailed data are given in Table 1.

\section{Surgical Data}

We compared surgical results and neurological outcomes between patients with aMNGs and pMNGs. The mean duration of surgery was 136 minutes in the aMNG group and 131 minutes in the pMNG group when the lesion was removed via the ULPA. When the lesion was removed via the BLPA, the mean duration of surgery was 224 minutes for aMNGs and 148 minutes for pMNGs. The main reasons for electing to conduct a BLPA were extensive calcifications and myelopathy demonstrated on the preoperative MRI. GTR, in accordance with Simpson grade II, was achieved in $93 \%$ of aMNGs when resected via a ULPA and in $92 \%$ of pMNGs when resected via a ULPA. In the group that required a BLPA, a Simpson grade II GTR was achieved in $94 \%$ of aMNGs and $96 \%$ of pMNGs. No significant difference was seen in resection grade when assessing the relationship of the MNGs to the denticulate ligament and surgical approach (Fig. 2B, Table 2). 

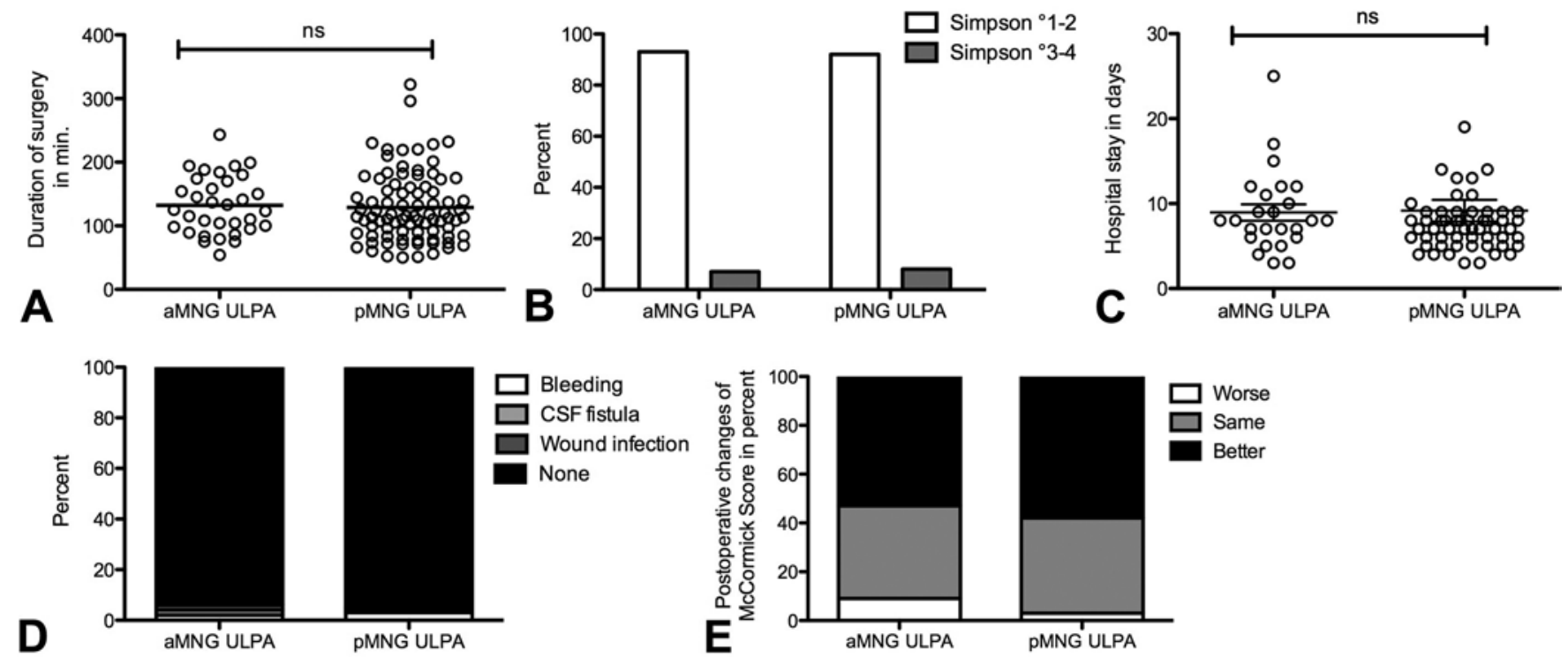

FIG. 2. A: Surgical duration in minutes (min.) according to approach and localization of the MNG. B: Extent of resection according to Simpson grade. C: Hospital stay in days according to the localization of the sMNG. D: Surgical complication rate according to localization of the MNG within the spinal canal. E: Changes in McCormick score before and after resection.

\section{Outcome and Follow-Up}

The majority of patients recovered quickly after surgery; the mean hospital stay was 9 days. Time to discharge did not vary significantly between groups (Fig. 2C). The surgical complication rate was $6 \%$ in patients with an aMNG treated via a ULPA and 5\% in those with a pMNG treated via a ULPA (Fig. 2D). In the group of patients

TABLE 2. Comparative data of aMNGs and pMNGs approached via a ULPA or BLPA

\begin{tabular}{|c|c|c|c|c|c|}
\hline \multirow[b]{2}{*}{ Variable } & \multicolumn{4}{|c|}{ Lesion Location \& Surgical Approach } & \multirow[b]{2}{*}{$\begin{array}{c}p \\
\text { Value }\end{array}$} \\
\hline & $\begin{array}{l}\text { aMNG } \\
\text { via } \\
\text { BLPA } \\
(n=16)\end{array}$ & $\begin{array}{l}\text { aMNG } \\
\text { via } \\
\text { ULPA } \\
(n=32)\end{array}$ & $\begin{array}{l}\text { pMNG } \\
\text { via } \\
\text { BLPA } \\
(n=25)\end{array}$ & $\begin{array}{l}\text { pMNG } \\
\text { via } \\
\text { ULPA } \\
(n=51)\end{array}$ & \\
\hline \multicolumn{6}{|l|}{ Mean size, $\mathrm{mm}$} \\
\hline Longitudinal & 19 & 19 & 16 & 18 & NS \\
\hline Sagittal & 11 & 12 & 12 & 10 & NS \\
\hline Axial & 13 & 12 & 11 & 11 & NS \\
\hline $\begin{array}{l}\text { Mean axial } \\
\text { invasion of the } \\
\text { spinal canal }\end{array}$ & $72 \%$ & $70 \%$ & $67 \%$ & $63 \%$ & NS \\
\hline $\begin{array}{l}\text { Duration of op, } \\
\text { mins }\end{array}$ & 224 & 136 & 148 & 131 & $<0.0001^{*}$ \\
\hline $\begin{array}{l}\text { Simpson grade II } \\
\text { resection }\end{array}$ & $94 \%$ & $93 \%$ & $96 \%$ & $92 \%$ & NS \\
\hline $\begin{array}{l}\text { Surgical complica- } \\
\text { tion rate }\end{array}$ & $6 \%$ & $6 \%$ & $8 \%$ & $5 \%$ & NS \\
\hline $\begin{array}{l}\text { Stable or improved } \\
\text { neurological } \\
\text { outcome }\end{array}$ & $88 \%$ & $97 \%$ & $92 \%$ & $99 \%$ & $0.0003 \dagger$ \\
\hline
\end{tabular}

* Calculated using the Bonferroni multiple comparison test.

$\dagger$ Calculated using the chi-square and Fisher exact tests. treated via a BLPA, the surgical complication rate was $6 \%$ in those with an aMNG and $8 \%$ in those with a pMNG. The overall surgical complication rate was 5\% and included CSF fistula $(n=2)$, bleeding $(n=7)$, and wound healing problems $(n=1)$. Neurological outcome was assessed at the time of discharge using the McCormick score and was compared to the presurgical status. Worsening of neurological function occurred in $3 \%$ of patients with an aMNG treated via the ULPA and in $1 \%$ of patients with a pMNG treated via the ULPA (Fig. 2E). In the group treated via the BLPA, neurological worsening occurred in $12 \%$ of patients with an aMNG and in $8 \%$ of patients with a pMNG (Table 2). In $72 \%$ of patients who showed impairment of neurological function after surgery, a complete recovery was shown at the time of follow-up 3 months after surgery.

Among patients who underwent a Simpson grade II GTR, the recurrence rate was $3.8 \%$ in those with WHO grade I MNGs and 9\% in those with WHO grade II MNGs. The median progression-free survival was 156 months. The progression-free survival rate at 10 years was $92 \%$. Risk factors for tumor recurrence $(\mathrm{n}=10)$ were incomplete resection $(\mathrm{n}=3)$ and WHO grade (WHO grade II [n = 1]); tumor localization had no impact on tumor recurrence.

\section{Illustrative Case}

Figure 3 shows a case of a 70-year-old woman who presented with back pain that had persisted for several weeks. Clinical examination showed no further neurological deficits. MRI revealed a contrast-enhancing mass within the spinal canal at the thoracolumbar junction. The tumor was located mainly anterior to the denticulate ligament with anterolateral expansion (Fig. 3A and B). The tumor was reached via a right-sided ULPA and was resected in piecemeal fashion. GTR was accomplished, and dura mater was coagulated as per specification of Simpson grade II. Histological examination revealed a WHO grade II atypi- 

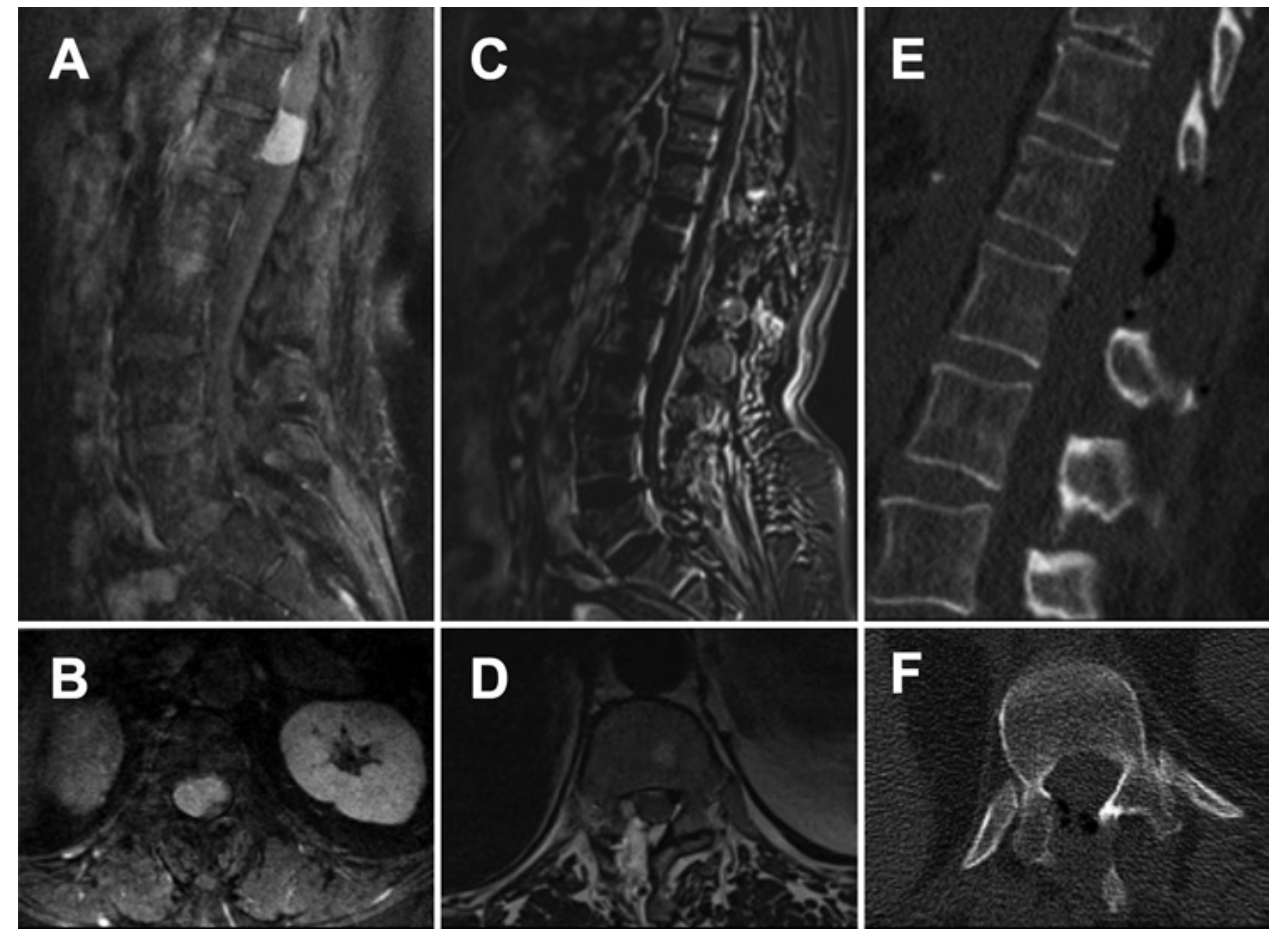

FIG. 3. Preoperative sagittal (A) and axial (B) MR images with contrast agent showing an aMNG at the thoracolumbar junction. Postoperative sagittal (C) and axial (D) MR images showing complete resection of the aMNG. Postoperative CT scans (E and F) demonstrating the extent of bony removal for a ULPA.

cal MNG. Postoperative MRI confirmed GTR of the tumor (Fig. 3C and D). The postoperative CT scans in Fig. $3 \mathrm{E}$ and $\mathrm{F}$ show the extent of bony removal achieved via ULPA. The patient recovered quickly after surgery and was discharged to home 8 days later without any neurological deficits.

\section{Discussion}

The principal novel finding of our study is that aMNG can be accessed and safely removed via a ULPA. The duration of surgery, resection grade, and outcome are comparable between aMNG and pMNG when removed via a ULPA. Thus, GTR with coagulation of the dura (Simpson grade II) is achievable in $>90 \%$ of cases and provides sufficient control of tumor recurrence, without the need to excise the dura.

In the literature, more advanced approaches, such as the posterolateral or combined approaches with dorsal instrumentation, have been described to access aMNG., ${ }^{9} 17$ In comparison to our results, the complication rates and access-related morbidity rates of these extended surgical approaches are high. ${ }^{1}$ To our knowledge, we report on the largest cohort of sMNGs that have been approached via a ULPA. With our data, we prove that a ULPA represents a suitable and safe approach even for sMNGs located anterior to the denticulate ligament, with a median surgical duration of 136 minutes and complete tumor removal in more than $92 \%$ of cases. Approaching aMNGs via a ULPA showed superior results to those that were removed via a BLPA, both in our own study and in the literature. ${ }^{12}$ This can be explained by the complexity of cases in the
BLPA group. In our study, a BLPA was chosen in cases that involved extensive tumor calcifications in combination with apparent myelopathy on the preoperative MRI. However, caution must be exercised in the interpretation of the data because different surgeons at two different institutions operated on heterogeneous cases. On the other hand, the comparison between outcomes of patients with aMNGs and those with pMNGs shows, despite different experience levels of participating surgeons, that there are no significant differences in surgical parameters and outcome in a well-balanced cohort (Table 1).

It is a matter of controversy of whether radical resection (Simpson grade I) is justified in sMNG due to the low recurrence rate associated with Simpson grade II and the increasing risk associated with radical surgical removal..$^{19}$ In this study, Simpson grade II resection was achieved in more than $90 \%$ of cases independent of tumor localization. Among these cases, the recurrence rate was low-3.8\% among those with WHO grade I MNGs-and comparable to those reported in the literature..$^{2,4,8,11,20}$

\section{Conclusions}

Duration of surgery, resection grade, and outcomes are comparable between aMNG and pMNG when removed via a ULPA. Thus, ULPA represents a safe method to achieve GTR, even in cases of aMNG. We conclude that Simpson grade II resection is a well-achievable therapeutic procedure, even from a ULPA, and that it provides low recurrence rates in WHO grade I MNGs. In complex cases with extensive tumor calcifications and myelopathy, more extended approaches are justified. 


\section{References}

1. Aboul-Enein HA, Khidr WM, Abdeen KM, Madawi AA: Surgical management of ventrally based lower cervical (subaxial) meningiomas through the lateral approach: report on 16 cases. Clin Neurol Neurosurg 139:152-158, 2015

2. Ambekar S, Sharma M, Kukreja S, Nanda A: Complications and outcomes of surgery for spinal meningioma: a Nationwide Inpatient Sample analysis from 2003 to 2010. Clin Neurol Neurosurg 118:65-68, 2014

3. Bydon M, Gokaslan ZL: Spinal meningioma resection. World Neurosurg 83:1032-1033, 2015

4. Durand A, Labrousse F, Jouvet A, Bauchet L, Kalamaridès $\mathrm{M}$, Menei P, et al: WHO grade II and III meningiomas: a study of prognostic factors. J Neurooncol 95:367-375, 2009

5. Gelabert González M, García Pravos A, Fernández Villa JM: [Spinal meningiomas.] Neurologia 15:58-62, 2000 (Span)

6. Goldbrunner R, Minniti G, Preusser M, Jenkinson MD, Sallabanda K, Houdart E, et al: EANO guidelines for the diagnosis and treatment of meningiomas. Lancet Oncol 17:e383e391, 2016

7. Gottfried ON, Gluf W, Quinones-Hinojosa A, Kan P, Schmidt $\mathrm{MH}$ : Spinal meningiomas: surgical management and outcome. Neurosurg Focus 14(6):e2, 2003

8. Kim CH, Chung CK, Lee SH, Jahng TA, Hyun SJ, Kim KJ, et al: Long-term recurrence rates after the removal of spinal meningiomas in relation to Simpson grades. Eur Spine J 25:4025-4032, 2016

9. Lonjon N, Russo V, Barbarisi M, Choi D, Allibone J, Casey A: Spinal cervical meningiomas: the challenge posed by ventral location. World Neurosurg 89:464-473, 2016

10. Louis DN, Perry A, Reifenberger G, von Deimling A, Figarella-Branger D, Cavenee WK, et al: The 2016 World Health Organization Classification of Tumors of the Central Nervous System: a summary. Acta Neuropathol 131:803-820, 2016

11. Maiti TK, Bir SC, Patra DP, Kalakoti P, Guthikonda B, Nanda A: Spinal meningiomas: clinicoradiological factors predicting recurrence and functional outcome. Neurosurg Focus 41(2):E6, 2016

12. Notani N, Miyazaki M, Kanezaki S, Ishihara T, Kawano M, Tsumura H: Surgical management of ventrally located spinal meningiomas via posterior approach. Eur J Orthop Surg Traumatol 27:181-186, 2017

13. Pompili A, Caroli F, Crispo F, Giovannetti M, Raus L, Vidiri A, et al: Unilateral laminectomy approach for the removal of spinal meningiomas and schwannomas: impact on pain, spinal stability, and neurologic results. World Neurosurg 85:282-291, 2016

14. Riad H, Knafo S, Segnarbieux F, Lonjon N: Spinal meningiomas: surgical outcome and literature review. Neurochirurgie 59:30-34, 2013

15. Setzer M, Vatter H, Marquardt G, Seifert V, Vrionis FD: Management of spinal meningiomas: surgical results and a review of the literature. Neurosurg Focus 23(4):E14, 2007
16. Sun SQ, Cai C, Ravindra VM, Gamble P, Yarbrough CK, Dacey RG, et al: Simpson grade I-III resection of spinal atypical (World Health Organization grade II) meningiomas is associated with symptom resolution and low recurrence. Neurosurgery 76:739-746, 2015

17. Takami T, Naito K, Yamagata T, Yoshimura M, Arima H, Ohata K: Posterolateral approach for spinal intradural meningioma with ventral attachment. J Craniovertebr Junction Spine 6:173-178, 2015

18. Tola S, De Angelis M, Bistazzoni S, Chiaramonte C, Esposito V, Paolini S: Hemilaminectomy for spinal meningioma: a case series of 20 patients with a focus on ventral- and ventrolateral lesions. Clin Neurol Neurosurg 148:35-41, 2016

19. Tsuda K, Akutsu H, Yamamoto T, Nakai K, Ishikawa E, Matsumura A: Is Simpson grade I removal necessary in all cases of spinal meningioma? Assessment of postoperative recurrence during long-term follow-up. Neurol Med Chir (Tokyo) 54:907-913, 2014

20. Westwick HJ, Shamji MF: Effects of sex on the incidence and prognosis of spinal meningiomas: a Surveillance, Epidemiology, and End Results study. J Neurosurg Spine 23:368-373, 2015

21. Yamamuro K, Seichi A, Kimura A, Kikkawa I, Kojima M, Inoue $\mathrm{H}$, et al: Histological investigation of resected dura mater attached to spinal meningioma. Spine (Phila Pa 1976) 37:E1398-E1401, 2012

\section{Disclosures}

Dr. Meyer reports being a consultant for Medtronic, DePuy, Icotec, Ulrich Medical, Brainlab, and Relievant. He has received support from Relievant, Icotec, and Medtronic for non-studyrelated clinical or research efforts. He receives royalties from Spineart.

\section{Author Contributions}

Conception and design: Vajkoczy, Onken, Meyer. Acquisition of data: Onken, Obermüller, Staub-Bartelt, Wostrack. Analysis and interpretation of data: Vajkoczy, Onken, Meyer, Wostrack. Drafting the article: Onken, Wostrack. Critically revising the article: Vajkoczy, Meyer, Wostrack. Reviewed submitted version of manuscript: Vajkoczy, Onken, Meyer, Wostrack. Statistical analysis: Onken. Administrative/technical/material support: Onken.

\section{Supplemental Information \\ Current Affiliations \\ Dr. Staub-Bartelt: University Hospital Düsseldorf, Germany.}

\section{Correspondence}

Peter Vajkoczy: Universitätsmedizin Charité Berlin, Germany. peter.vajkoczy@charite.de. 